\title{
Hepatic Fibrosis Assessment Using Fractal Analysis as a Histological Grading Method
}

\author{
Claudiu GAL, Liviu GAIȚĂ, Claudia CONSTANTINESCU, Livia DUMITRESCU, Manuella MILITARU \\ Faculty of Veterinary Medicine, University of Agronomic Sciences and \\ Veterinary Medicine of Bucharest, 105 Splaiul Independenței, 050097, Bucharest, Romania \\ gal.claudiu@gmail.com
}

Bulletin UASVM Veterinary Medicine 73(1)/2016

Print ISSN 1843-5270; Electronic ISSN 1843-5378

doi:10.15835/buasvmcn-vm: 11379

\begin{abstract}
The histopathologic examination of hepatic fibrosis remains the gold standard in routine diagnosis of hepatopathies in veterinary medicine. However, the visual evaluation of fibrosis is usually limited by the observer's capabilities. This paper aimed to present the applicability of fractal analysis as a quantitative method for evaluating the liver fibrosis grading using accessible tools, compared to one of the semiquantitative methods used currently in human histopathology, the METAVIR scoring system. Ovine liver histological sections taken from 22 slaughtered lambs, Masson's trichrome-stained, were analyzed using the METAVIR score as the semiquantitative method for staging the liver fibrosis and inflammatory reaction, and the fractal dimension of collagen regions as a quantitative method for fibrosis evaluation. The results show strong correlations between fibrosis and fractal dimension as the statistics revealed that there is a significant difference between the presence of fibrosis and its absence $(\mathrm{p}<0.001)$. Significant differences between the fractal dimensions corresponding to F1 and F2 fibrosis grades were observed for the images aquired with objective lens $20 \times$. The presence of inflammatory cells did not influenced the fractal dimension of fibrosis. The Receiver Operating Characteristic (ROC) plot provided fair results for discriminating F1 versus F2 fibrosis grades, with the area under the ROC curve of 0.79 . The study showed significant differences between the fractal dimension values of the liver tissue with different fibrosis grades, therefore, the fractal analysis provides a useful complementary tool in the histological examination.
\end{abstract}

Keywords: digital imaging, fractal analysis, hepatic fibrosis, METAVIR staging

\section{INTRODUCTION}

The tendency of quantifying the observed phenomena comes as a logical evolution from the simple qualitative or classificatory observation and semi-quantitative measurements (Dioguardi et al., 2005). Considering the development of the virtual microscopy and digital image analysis, the need to find an unbiased evaluation of the histological elements found in fibrosis led to new methods for staging fibrosis that have the potential of becoming reliable tools in the quantitative appreciation of architectural alteration of a tissue (Dioguardi, 2011; Grizzi et al., 2006). The state of the art in digital image analysis of liver fibrosis includes digital slide scanning and automated image processing and morphometry, intending to become a "virtual expert pathologist" (Calès et al., 2015). Campos et al. (2014) proposed a more affordable method for liver fibrosis quantification by measuring the area of fibrosis using an inexpensive and commonly available imaging software as a complementary tool for the traditional histological methods. Our study aimed to explore the efficacy of fractal analysis in identifying the presence and the complexity of fibrous tissue in liver fibrosis using accessible tools on micrographs of Masson trichrome stained histological sections.

In the liver fibrosis, the irregular morphology of the collagen elements and their susceptibility to change shape and size depending on the scale at which they are observed determine only approxi- 
mate measures using the Euclidean geometry methods (Dioguardi, 2005). The extra-cellular matrix (ECM) deposition in liver fibrosis, through its irregular shape, would rather be described as a fractal object with an associated noninteger dimension. Previous studies explored the possibility of using fractal analysis to characterize the liver fibrosis, proving this method to be an accurate quantitative morphometric measurement of the geometric complexity of the connective tissue. Also, the technique has been described as reproducible, simple, and adaptable. (Grizzi et al., 2006; Moal, 2002)

As a model for studying fibrotic changes, the liver of sheep naturally infected with the lancet liver fluke (Dicrocoelium dendriticum) proved to be a convenient source of fibrous lesions, as a recent study evince (Kukolj et al., 2015). The main lesions determined by lancet liver flukes are bile ducts hyperplasia, portal or periportal hepatitis, and mild to severe hepatic fibrosis (Manga-González et al., 2004).

\section{MATERIALS AND METHODS}

For this study, two liver samples from each of the 22 slaughtered lambs diagnosed with dicrocoeliasis were histopathologically processed. A section of 4-5 $\mu \mathrm{m}$ thickness from each formalin-fixed, paraffin-embedded tissue sample was Masson trichrome stained for collagen depositions and examined. Images were acquired using an Olympus BX41 microscope equipped with Olympus SP350 video camera and the Cell ${ }^{\wedge} \mathrm{B}$ software and saved as 24 bits RGB images in the tagged image file format (TIFF) (Cromey, 2010). Micrographs were captured at a resolution of 300 dpi with $2560 \times 1920$ pixels using the objective lenses (ob.) of $4 \times, 10 \times, 20 \times$ and $40 \times$. For a broader view some images $(n=27)$ were captured with ob. $4 \times$ and assembled with the multiple image alignment (MIA) technique available in the $\mathrm{Cell}^{\wedge} \mathrm{B}$ software.The digital processing was carried out with the Corel $^{\circledR}$ Photo-Paint ${ }^{\mathrm{m}}$ X7 software (Corel Company, Ottawa, Ontario, Canada) and consisted in two methods. The first approach comprised the increasing by $20 \%$ of the contrast in the "Brightness/Contrast/Intensity" dialog box and the colour saturation of the master channel by $30 \%$, in "Hue/Saturation/Lightning” dialog box from the Adjust menu, and the directional sharpness of the micrograph by $100 \%$ from the Effects - Sharpen menu, for a better identification of the fibrous tissue. Using the replace colour function with the option of single destination colour, the blue shades of collagen fibers were replaced with a uniform red colour with full saturation and a range of 40, followed by the applying of a colour mask for red. After inverting the mask, the rest of the image that wasn't red was cropped out. In some images, even though the fibrous tissue seemed of a distinct colour than the parenchyma, the software could not identify it in the colour mask application but a manual colour picking improved the outcome. As a final step in the image preparation for the fractal analysis, a black and white (1 bit) conversion was made into a TIFF image with the resolution of 300 dpi with $800 \times 600$ pixels using the line art method with a threshold of 130 (Fig. 1).

The second method consisted in a batch processing of all images using a recorded script. The script included the increase by $20 \%$ of the contrast and by $100 \%$ of the directional sharpness from the same dialog boxes as described in the previous method. From the „Hue/Saturation/
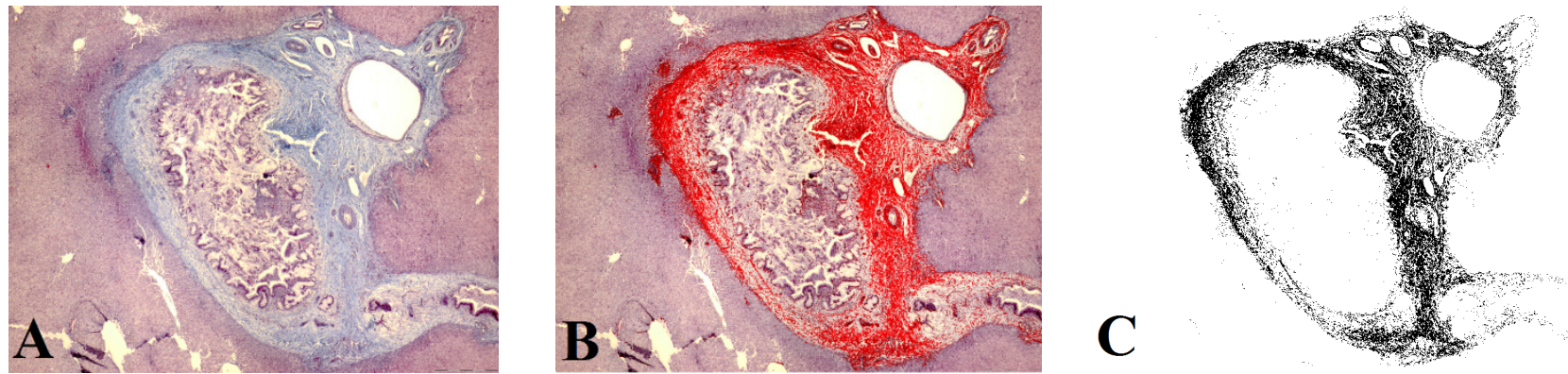

Fig. 1. The first method of image processing: the micrograph with enhanced contrast, colour saturation and sharpness (A), red mask applied over the collagen fibers (B) and the black and white (1-bit) image of the extracted fibrous tissue (C) 
Lightning" dialog box the saturation value of the red and magenta channels was set to -100 , for shifting the red cells and hepatocytes colour to grey, and the saturation for the blue channel was set to 100 . The colour mask was applied only for blues, selecting the blue color with Hex value of $\# 0000 \mathrm{FF}$, setting the smooth value to 100 , the tolerance to HSB mode, with Hue value set to 10, Saturation value set to 100 , Brightness value set to 100 and Threshold set To Black with value set to 0 . After inverting the mask and cropping out all the elements except the blue ones, the image was converted to grayscale (8-bit), using the default grayscale colour profile with dot gain set to $15 \%$, into TIFF images with the resolution of $300 \mathrm{dpi}$ with $1024 \times 768$ pixels (Fig. 2). The images in which the fibrous tissue couldn't be automatically isolated from the parenchyma $(n=47)$ were eliminated from further usage.

For the semi-quantitative estimation of fibrosis grade, the method used in this study is represented by the METAVIR score (Meta-analysis of Histological
Data in Viral Hepatitis), developed by the french METAVIR cooperative group, which consists of two variables, $\mathrm{F}$ representing the fibrosis estimation and A representing the necroinflammatory features (Bedossa et al., 1996). The fibrosis presence was classified as F0 for the absence of fibrosis, F1 for the fibrous portal expansion, F2 for few bridges or septa, F3 for numerous bridges or septa and F4 for cirrhosis. The presence of the inflamatory cells, described in Table 1, can alter the visibility of the ECM architecture affecting thus the fractal dimension of the image.

From the two different processing methods resulted two sets of fractal dimension values labeled M1 and M2, which were grouped according to the fibrosis degree expressed in the METAVIR score and the magnification of the objective lenses. For verifying if the presence of inflammatory reaction influences the fractal dimension of fibrous tissue, another subgrouping by the METAVIR necroinflammatory score was created in each of these groups.
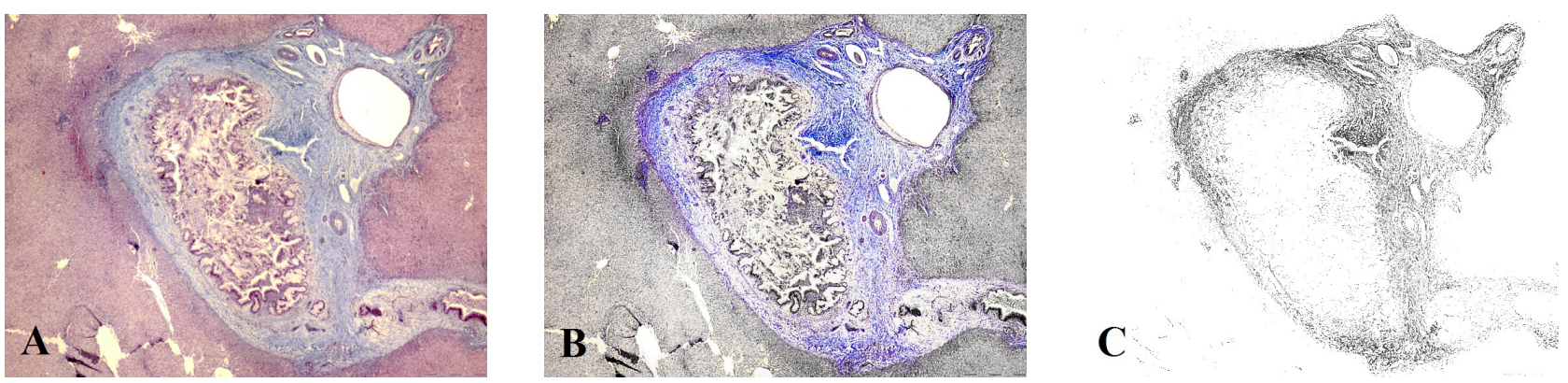

Fig. 2. The second method of image processing: the original micrograph (A), oversaturation of blue and desaturation of red and magenta colours (B) and the grayscale (9-bit) image of the extracted fibrous tissue (C)

Tab. 1. Necroinflammatory presence classification (Bedossa et al., 1996)

\begin{tabular}{|l|l|}
\hline \multicolumn{2}{|c|}{ The necroinflammatory reaction (A) } \\
\hline A0 = PMN0, LN0 & Piecemeal necrosis (PMN) = \\
- absent & - - absent; \\
\hline A1 = PMN0, LN1 & - mild, with focal alteration of the periportal plate in some portal tracts; \\
PMN1, LN0/1 & 2- moderate, diffuse alteration of the periportal plate in some \\
- mild & portal tracts or focal lesions around all portal tracts; \\
\hline A2 = PMN0, LN2 & - - severe, diffuse alteration of the periportal plate in all portal tracts \\
PMN1, LN2 & Focal lobular necrosis (LN) = \\
PMN2, LN0/1 & 0 - less than one necroinflammatory foci per lobule; \\
- moderate & 1- at least one necroinflammatory foci per lobule; \\
A3 = PMN2, LN2 & 2- several necroinflammatory foci per lobule \\
PMN3, LN0/1/2 & or confluent or bridging necrosis \\
- severe & \\
\hline
\end{tabular}


The box-counting method, which proved to be by far the most reliable (Losa, 2015), was used to calculate the fractal dimension $\left(\operatorname{dim}_{b o x}(S)\right)$, as defined in Eq.(1) where $N$ is the number of boxes of side length $(\varepsilon)$ required to cover the set $(S)$.

$$
\operatorname{dim}_{b o x}(S)=\lim _{\varepsilon \rightarrow 0} \frac{\log N(\varepsilon)}{\log (1 / \varepsilon)}
$$

The fractal dimension was determined for the ECM regions extracted through digital processing using the FracLab 2.0 software, developed by Research Center INRIA Saclay - Île-de-France. It was computed using the box method for signal or gray scale image, using the regression curve drawn by the least squares method. The technique has been adapted from previous studies on fractal analysis of chromatin regions in epithelial tumours (Gaiță, 2013). The more detailed settings are shown in figure 2 .

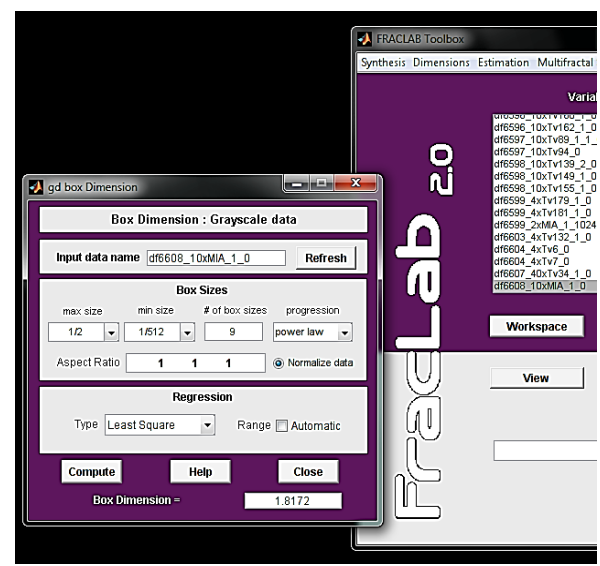

Fig. 3. Fraclab 2.0 settings used in the fractal dimension computation

The StatsDirect 3.0 software was used for the statistical analysis and for creating the Box and Whisker plots. The standard normal distribution of the fractal dimension values was verified using the Skewness, Kurtosis, Royston $\chi^{2}$, Shapiro-Wilk, Shapiro-Francia $\mathrm{W}$ tests. For the sample groups with unequal sizes and normal distribution a one way analysis of variance was performed using the Tukey-Kramer test that allows successive multiple comparisons of the means of each sample group. The non-parametric Mann-Whitney U test was used in the comparison of samples unlikely to be from a normal distribution. The statistically significant level was defined at $p<0.05$ for all comparisons, assuming one-tailed tests in comparing groups of different fibrosis grade and twotailed tests in comparing subgroups of different necroinflammatory score.

To verify the usefulness of fractal analysis in the quantitative fibrosis evaluation, the Receiver Operating Characteristic (ROC) test was used.

\section{RESULTS}

The histopathological findings included typical lesions for the lancet liver fluke infestation. The bile ducts hyperplasia usually coexisted with periductal fibrosis and occasionally with leukocytic infiltration. Hepatic fibrosis was mostly limited to the periportal spaces, sometimes extended to septal fibrosis, rarely forming fibrous bridges. Flukes covered in mucus were infrequently detected in the bile ducts, accompanied by severe epithelial hyperplasia, fibrosis and mononuclear inflammatory cells.

In the METAVIR evaluation each micrograph received a score of F0 ( $\mathrm{n}=30), \mathrm{F} 1 \quad(\mathrm{n}=90)$ or F2 $(n=107)$. The fibrosis score exceeded the F2 level only twice in the examined samples so the images graded F3 were excluded from statistic analysis because of insufficient data. Often, the stages F1 and F2 were difficult to distinguish because of the discreet interlobular septa. Also, the necroinflammatory reaction in the hepatic lobules was rarely observed.

With the use of Box and Whisker plots, the fractal dimension values were represented graphically for each objective lens group in the two sets corresponding to the methods of digital processing and for each sample of fibrosis grade with its size $n$ (Fig. 4-11). Due to small sample sizes the fractal dimension values for images acquired with ob. 40× were excluded from analysis.

From the two sets of data, M1 and M2, most of the group samples followed a normal distribution except F2 ob. 10× from M1 and F1 ob.10× from M2. In the METAVIR necroinflammatory score subgroups, the tests revealed no patterns of nonnormality in most of the samples. The fractal dimension values from A0 F0 ob. 4×, A1 F1 ob. 4×, A2 F1 ob. $10 \times$, and A1 F2 ob. $10 \times$ groups of the M1 set were unlikely to be from a normal distribution. Other small size samples that could not be verified with any of the applied tests were not used in further statistical comparisons. 


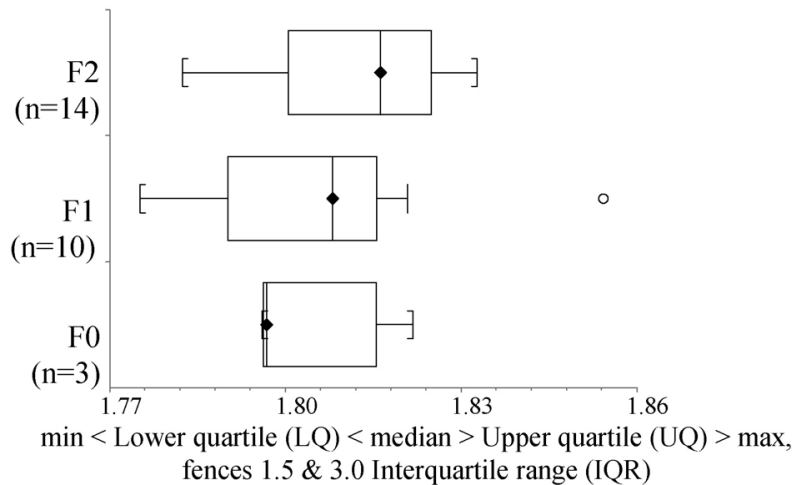

Fig. 4. Fractal dimensions for MIA group, M1

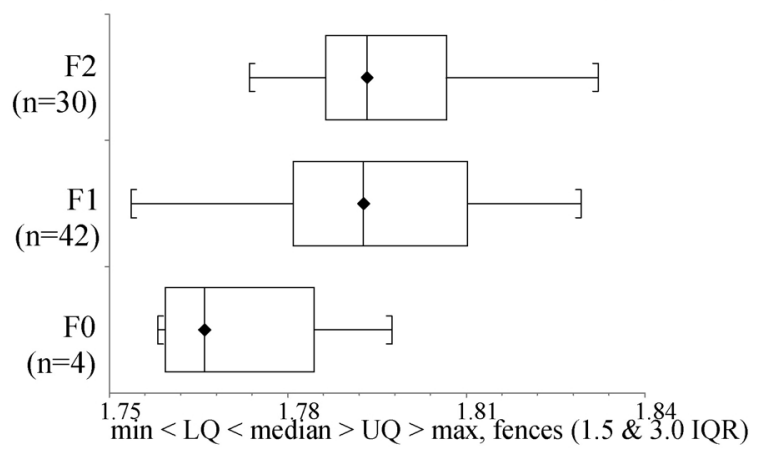

Fig. 6. Fractal dimensions for ob. $10 \times$ group, M1

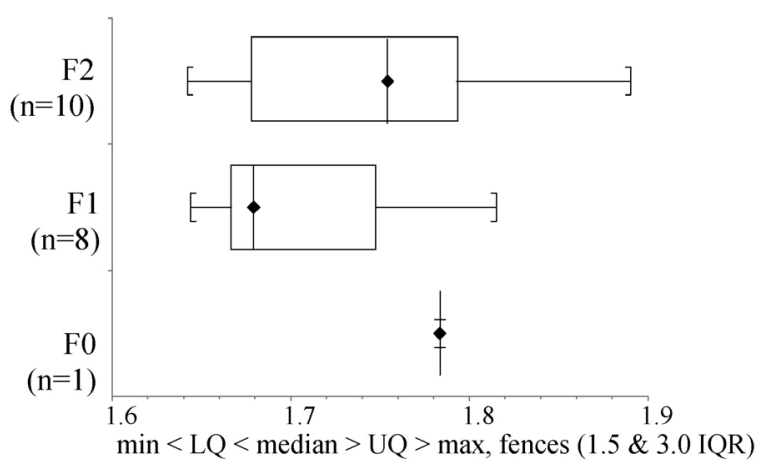

Fig. 8. Fractal dimensions for MIA group, M2

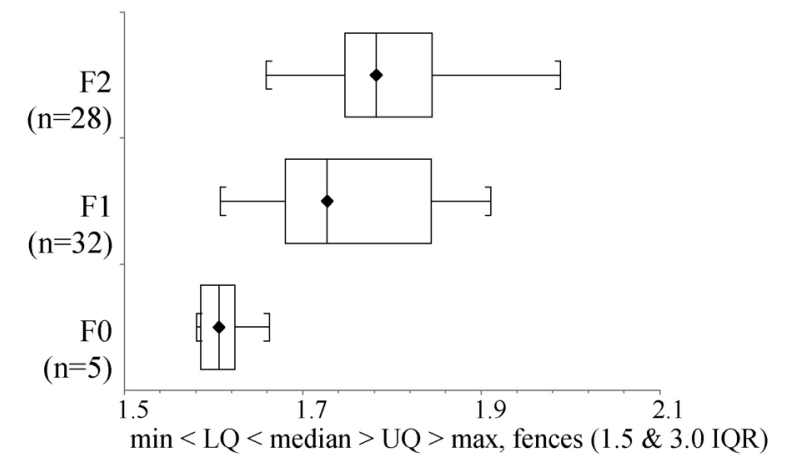

Fig. 10. Fractal dimensions for ob. $10 \times$ group, M2

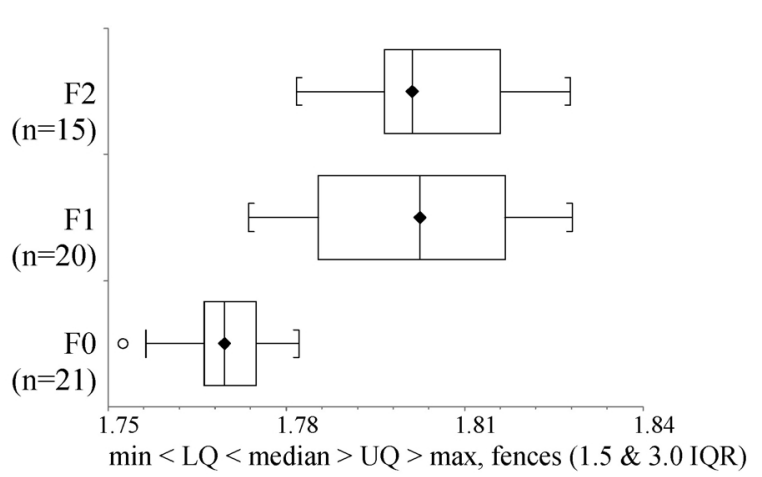

Fig. 5. Fractal dimensions for ob. $2 \times$ group, M1

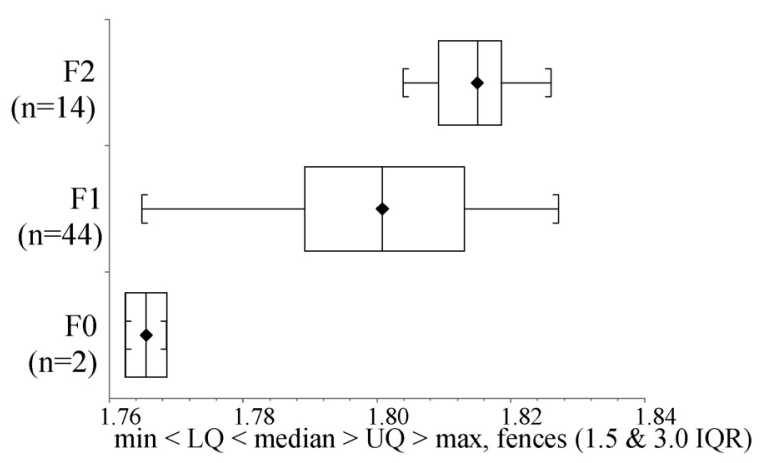

Fig. 7. Fractal dimensions for ob. $20 \times$ group, M1

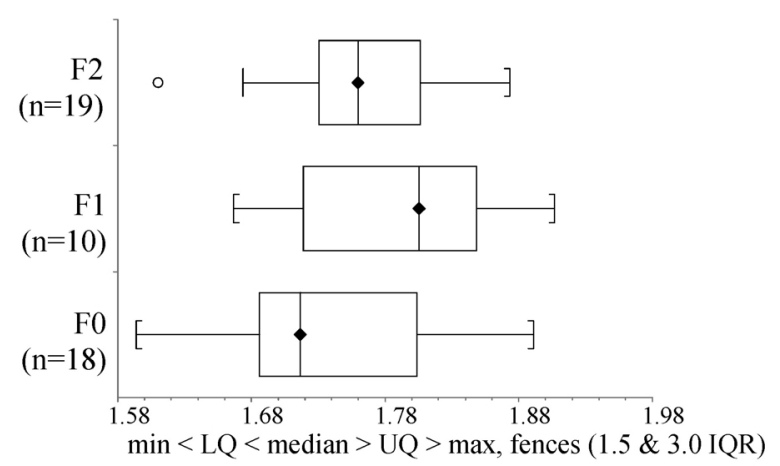

Fig. 9. Fractal dimensions for ob. $2 \times$ group, M2

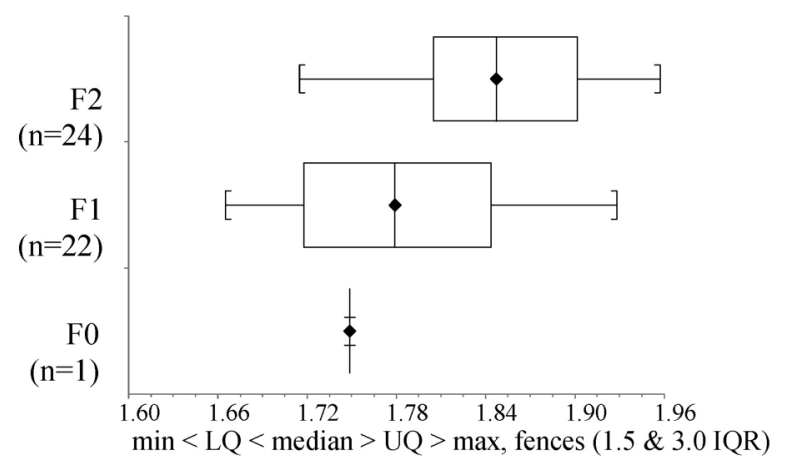

Fig. 11. Fractal dimensions for ob. $20 \times$ group, M2 
According to the Tukey-Kramer multiple comparison tests used for the samples with normal distribution there are statistically significant differences especially between the groups of fractal dimension values corresponding to images with and without fibrosis (Table 2).
The Mann-Whitney U tests applied to samples unlikely to have a normal distribution indicated in the compared groups that the fractal dimension values of images with no fibrosis tend to be smaller than those of images with fibrosis for both digital processing methods and in the second method being able to indicate also that the fractal dimension

Tab. 2. The Tukey-Kramer test results of fractal dimension values comparisons specific to fibrosis grade samples. The statistically significant contrasts are highlighted.

\begin{tabular}{|c|c|c|c|c|}
\hline Set & Comparison & Mean difference & Confidence interval 95\% & $p$ value \\
\hline \multirow{5}{*}{ M1 } & F0 ob $4 \times$ vs. F2 ob $4 \times$ & -0.034699 & $(-0.044984$ to -0.024414$)$ & $<0.0001$ \\
\hline & F0 ob $4 \times$ vs. F1 ob $4 \times$ & -0.030976 & $(-0.040481$ to -0.02147$)$ & $<0.0001$ \\
\hline & F1 ob4× vs. F2 ob4× & -0.003723 & $(-0.014115$ to 0.006668$)$ & 0.6652 stop \\
\hline & F0 ob10× vs. F1 ob10× & -0.019602 & $(-0.033269$ to -0.005934$)$ & 0.0058 \\
\hline & F1 ob20x vs. F2 ob20x & -0.033264 & $(-0.059379$ to -0.007148$)$ & 0.0092 \\
\hline \multirow{5}{*}{ M2 } & F0 ob4× vs. F2 ob4× & -0.051872 & $(-0.127813$ to 0.024068$)$ & 0.2332 stop \\
\hline & F0 ob $4 \times$ vs. F1 ob $4 \times$ & -0.029888 & $(-0.093219$ to 0.033443$)$ & 0.4922 \\
\hline & F1 ob $4 \times$ vs. F2 ob4× & 0.021984 & $(-0.053239$ to 0.097208$)$ & 0.7596 \\
\hline & F0 ob10x vs. F2 ob10x & -0.187981 & $(-0.256136$ to -0.119825$)$ & $<0.0001$ \\
\hline & F1 ob20× vs. F2 ob20x & -0.031186 & $(-0.206938$ to 0.144566$)$ & 0.9032 \\
\hline
\end{tabular}

Tab. 3. The Mann-Whitney U test results of fractal dimension values comparisons specific to fibrosis grade samples. The statistically significant contrasts are highlighted.

\begin{tabular}{ccccccc}
\hline Set & Groups compared & $n$ & Median & Rank sum & Uvalue & Lower side $p$ value \\
\hline \multirow{3}{*}{ M1 } & F0 ob10x & 4 & 1.79275 & 29 & \multirow{2}{*}{0.0131} \\
\cline { 2 - 7 } & F2 ob10x & 30 & 1.79335 & \multirow{2}{*}{1468.5} & \multirow{2}{*}{565.5} & \multirow{2}{*}{0.2327} \\
\hline \multirow{3}{*}{ F1 ob10x } & 42 & 1.79275 & 20 & 5 & $<0.0001$ \\
\cline { 2 - 7 } & F2 ob10x & 30 & 1.79335 & 1.6059 & \multirow{2}{*}{307.5} & 0.0185 \\
\hline
\end{tabular}

Tab. 4. The Tukey-Kramer test results of fractal dimension values comparisons corresponding to the necroinflammatory score samples specific to a fibrosis grade.

\begin{tabular}{|c|c|c|c|c|}
\hline Set & Comparison & Mean difference & Confidence interval 95\% & $p$ value \\
\hline \multirow{9}{*}{ M1 } & A0 F1 ob. $4 \times$ vs. A2 F1 ob. $4 \times$ & -0.008713 & $(-0.039658$ to 0.022232$)$ & 0.5166 stop \\
\hline & A1 F2 ob. $4 \times$ vs. A2 F2 ob. $4 \times$ & 0.00745 & $(-0.006855$ to 0.021755$)$ & 0.2786 stop \\
\hline & A0 F1 ob. $10 \times$ vs. A1 F1 ob. $10 \times$ & -0.002534 & $(-0.02249$ to 0.017421$)$ & 0.7979 stop \\
\hline & A1 F1 ob. $20 \times$ vs. A2 F1 ob. $20 \times$ & 0.01159 & $(-0.010085$ to 0.033265$)$ & 0.4032 stop \\
\hline & A0 F1 ob. $20 \times$ vs. A2 F1 ob. $20 \times$ & 0.01403 & $(-0.013519$ to 0.041579$)$ & 0.4378 \\
\hline & A0 F1 ob. $20 \times$ vs. A1 F1 ob. $20 \times$ & 0.00244 & $(-0.017194$ to 0.022074$)$ & 0.951 \\
\hline & A1 F2 ob. $20 \times$ vs. A2 F2 ob. $20 \times$ & 0.009017 & $(-0.004558$ to 0.022592$)$ & 0.2165 stop \\
\hline & A0 F2 ob. $20 \times$ vs. A1 F2 ob. $20 \times$ & 0.00795 & $(-0.007902$ to 0.023802$)$ & 0.3964 \\
\hline & A0 F2 ob. $20 \times$ vs. A2 F2 ob. $20 \times$ & -0.001067 & $(-0.012643$ to 0.01051$)$ & 0.9665 \\
\hline \multirow{10}{*}{ M2 } & A0 F1 ob. $4 \times$ vs. A1 F1 ob. $4 \times$ & 0.090792 & $(-0.019684$ to 0.201268$)$ & 0.0947 stop \\
\hline & A0 F2 ob. $4 \times$ vs. A2 F2 ob. $4 \times$ & -0.119767 & $(-0.304855$ to 0.065322$)$ & 0.2468 stop \\
\hline & A1 F2 ob. $4 \times$ vs. A2 F2 ob. $4 \times$ & -0.0989 & $(-0.277256$ to 0.079456$)$ & 0.3493 \\
\hline & A0 F2 ob. $4 \times$ vs. A1 F2 ob. $4 \times$ & -0.020867 & $(-0.106546$ to 0.064813$)$ & 0.8068 \\
\hline & A0 F1 ob. $10 \times$ vs. A1 F1 ob. $10 \times$ & -0.011848 & $(-0.080367$ to 0.05667$)$ & 0.7264 stop \\
\hline & A1 F2 ob. $10 \times$ vs. A2 F2 ob. $10 \times$ & 0.071747 & $(-0.013313$ to 0.156806$)$ & 0.11 stop \\
\hline & A0 F1 ob. $20 \times$ vs. A2 F1 ob. $20 \times$ & 0.04864 & $(-0.076093$ to 0.173373$)$ & 0.5915 stop \\
\hline & A0 F1 ob. $20 \times$ vs. A1 F1 ob. $20 \times$ & 0.032668 & $(-0.07231$ to 0.137647$)$ & 0.7132 \\
\hline & A1 F1 ob. $20 \times$ vs. A2 F1 ob. $20 \times$ & 0.015972 & $(-0.089007$ to 0.12095$)$ & 0.9213 \\
\hline & A0 F2 ob. $20 \times$ vs. A1 F2 ob. $20 \times$ & 0.02835 & $(-0.042308$ to 0.099008$)$ & 0.5781 \\
\hline
\end{tabular}


values of images graded F1 are smaller than those of images graded F2, as shown in Table 3.

In the comparisons of necroinflammatory score subgroups the Tukey-Kramer tests revealed no significant contrast between the samples ( $p$ $>0.5$ ) (Table 4), confirming the null hypothesis that the values from compared subgroups are not different. As shown in Table 5, the Mann-Whitney $\mathrm{U}$ tests also indicated no statistically difference between the compared subgroups ( $p>0.5$, twotailed).

Considering the Box and Whisker plots and the statistical comparisons, ROC curve analyses were performed for the groups F1 and F2 with statistically significant differences, in order to assess the possibility of fractal analysis usage in identifying the fibrosis grade by considering $\mathrm{F} 1$ as the absence of the disease and F2 as the presence of the disease. For the ob. 10x group of M2 set two additional curves were drawn for $\mathrm{F} 0$ as the absence of the disease and F1, respectively F2, as the presence of the disease (Fig. 12-14).

The ROC plots revealed a powerful discrimination ability between healthy tissue and fibrosis of any degree reporting an area under the receiver operating characteristic (AUROC) over 0.95. For the fibrosis grades distinction the test had a fair performance only for the images acquired with objective lens $20 \times$ (Table 6).

\section{DISCUSSION}

Although the digital image analysis has reached high levels of performance using multiple parameters and cutting edge tools (Calès et al., 2015), there is still a need for affordable and reliable techniques that can be used especially in the research field. The present study proposed the usage of fractal dimension as a unique descriptor for the fibrosis grade. Other inexpensive quantifying methods rely mainly on counting the specific pixels found in the fibrosis area (Campos et al., 2014). Some authors (Dioguardi, 2005, Grizzi et al. 2006) state that the disadvantage of sole metric measurements of collagen area is that the measures reflects only the state of that section and do not fully represent the status of the organ as a whole and recommend the additional usage of fractal dimension as a correction parameter.

Attaining fibrosis lesions from the liver of sheep naturally infected with the lancet liver fluke (Kukolj et al., 2015) is more cost efficient and bureaucratically facile than inducing experimental hepatic fibrosis. The disadvantage lies within not being able to control the variability of the lesions encountered.

Tab. 5. The Mann-Whitney U test results of fractal dimension values comparisons corresponding to the necroinflammatory score samples specific to a fibrosis grade.

\begin{tabular}{|c|c|c|c|c|c|c|}
\hline Set & Subgroups compared & $n$ & Median & Rank sum & $U$ value & Two sided $p$ value \\
\hline \multirow{6}{*}{ M1 } & $\begin{array}{l}\text { A0 F0 ob4× } \\
\text { A1 F0 ob4x }\end{array}$ & $\begin{array}{c}16 \\
5\end{array}$ & $\begin{array}{l}1.76905 \\
1.7748\end{array}$ & 170 & 34 & 0.6459 \\
\hline & $\begin{array}{l}\text { A0 F1 ob4x } \\
\text { A1 F1 ob4x }\end{array}$ & $\begin{array}{c}3 \\
12\end{array}$ & $\begin{array}{l}1.7877 \\
1.8099\end{array}$ & 12 & 6 & 0.1011 \\
\hline & $\begin{array}{l}\text { A1 F1 ob4x } \\
\text { A2 F1 ob4× }\end{array}$ & $\begin{array}{c}12 \\
5\end{array}$ & $\begin{array}{l}1.8099 \\
1.7863\end{array}$ & 116 & 38 & 0.4421 \\
\hline & $\begin{array}{l}\text { A0 F1 ob10x } \\
\text { A2 F1 ob10x }\end{array}$ & $\begin{array}{l}5 \\
6\end{array}$ & $\begin{array}{l}1.7942 \\
1.8039\end{array}$ & 29 & 14 & 0.9307 \\
\hline & $\begin{array}{l}\text { A1 F1 ob10x } \\
\text { A2 F1 ob10x }\end{array}$ & $\begin{array}{c}31 \\
6\end{array}$ & $\begin{array}{l}1.7873 \\
1.8039 \\
\end{array}$ & 583 & 87 & 0.8252 \\
\hline & $\begin{array}{l}\text { A1 F2 ob10x } \\
\text { A2 F2 ob10x }\end{array}$ & $\begin{array}{l}18 \\
10\end{array}$ & $\begin{array}{l}1.79425 \\
1.79015 \\
\end{array}$ & 287 & 116 & 0.2259 \\
\hline
\end{tabular}

Tab. 6. ROC tests results. Excellent accuracy in discriminating F0 vs. F1 or F2 fibrosis degree and a fair performance in differentiating F1 and F2 in images captured with ob. 20x

\begin{tabular}{cccccc}
\hline Set & Samples & Sensitivity & Specificity & Cutt-off value & AUROC \\
\hline M1 & F1 ob. $20 \times$ vs. F2 ob. $20 \times$ & 1 & 0.613 & 1.8039 & 0.76 \\
\hline & F0 ob. $10 \times$ vs. F1 ob. $10 \times$ & 0.875 & 1 & 1.6731 & 0.96 \\
\cline { 2 - 6 } M2 & F0 ob. $10 \times$ vs. F2 ob. $10 \times$ & 0.964 & 1 & 1.7102 & 0.99 \\
\cline { 2 - 6 } & F1 ob. $10 \times$ vs. F2 ob. $10 \times$ & 0.821 & 0.562 & 1.737 & 0.65 \\
\cline { 2 - 6 } & F1 ob. $20 \times$ vs. F2 ob. $20 \times$ & 0.755 & 0.59 & 1.801 & 0.73 \\
\hline
\end{tabular}




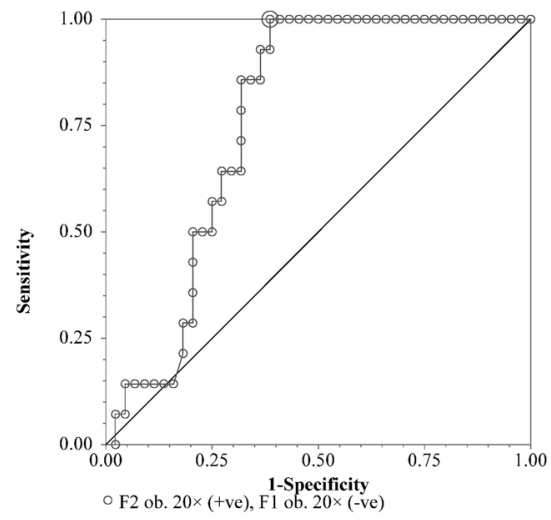

Fig. 12. ROC plot for fibrosis grades F1 and F2 of ob. $20 \times$, M1 set

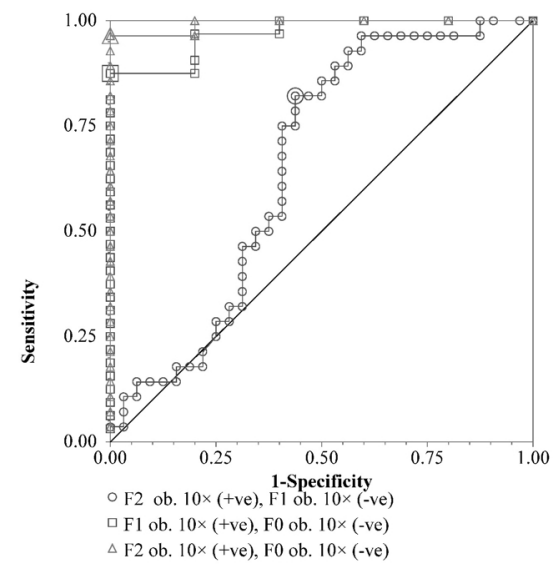

Fig. 13. ROC plot for fibrosis grades F0, F1 and F2 of ob. 10×, M2 set

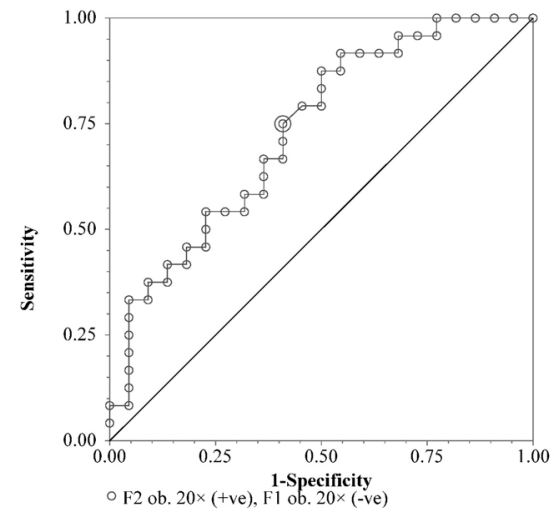

Fig. 14. ROC plot for fibrosis grades $F 1$ and F2 of ob. $20 \times$, M2 set

Depending on the stage of the disease and the host response, the bile ducts irritation and the toxic effect of metabolic products produced by the parasite may cause various lesions, from hyperplasia of bile duct epithelium and periductal inflammation to portal and septal fibrosis. Considering the etiology and the young age of the lambs, the histological findings were similar to another study (Manga-González et al., 2004). The limitation of the fibrous tissue around the periportal spaces and the scarcity of the fibrous septa reflected in the discreet differences of fractal dimensions between the METAVIR scores F1 and F2, influencing the statistical analysis through the small variability in collagen architecture.

The tissue samples were larger than those resulted from biopsy, as used in other studies (Calès et al., 2015, Dioguardi, 2005), so it allowed the aquiring of more images from the same sample. The aim of the image selection was to evaluate the efficiency of fractal analysis used in hepatic fibrosis reckoning and not to describe the lesions of the entire organ, thus the number of sections from each liver is not relevant but the number of micrographs showing different stages of fibrosis.

As Campos et al. (2014) affirmed, even though most of the studies on quantitative liver fibrosis consider the Sirius Red as the best technique for staining collagen, the Masson Trichrome staining is more common and offers a fair contrast that proved to be adequate for the present study.

The digital image analysis technique presented in this article attempts to be a low-cost method that provides a quantitative evaluation of liver fibrosis without the use of expensive glass slide scanning devices and third party software. The Core $^{\circledR}{ }^{\circledR}$ Photo-Paint ${ }^{\mathrm{m}} \mathrm{X7}$ software, as a commonly used image processing software, proved to be a reliable and accessible tool that could be used according to the guidelines suggested by Cromey (2010) regarding the manipulation of scientific digital images. The images processing was realised 
by two methods, one partially manually and one automated. The first one consisting in a mild adjustment of contrast and saturation, yielded a monochrome image similar to the digitized patterns of the collagen tissue used in other studies (Grizzi et al., 2006; Moal, 2002), reflecting the spread of the collagen fibers but with few details regarding the fibers texture. The second method was performed in batch after recording a script of processes for the first analysed image, using the recorder docker, and then, applying the script to all images, therefore reducing considerably the time consumed. In this method the oversaturation was not done to such extent that the blue pixels would turn white when converting to grayscale as Cromey (2010) apprise.

The fractal analysis was able to perfectly differentiate between the presence or the absence of fibrosis $(p<0.0001)$ in images processed with the first method and captured with $4 \times$ or $10 \times$ objective lenses. Not being able to distinguish the presence of fibrosis in images acquired with ob.4x and processed with the second method suggest the scanty details captured at such a low magnification and their loss in image processing. This fact explains also the inability of both processing methods to distinguish between F1 and F2 fibrosis grades in images captured with ob. $4 \times$. As the objective lens power increased at $20 \times$, the ability to discriminate between F1 and F2 fibrosis grades improved, both methods having a fair performance in the ROC analysis $(0.7<$ AUROC $<0.8)$ and a cut-off level of 1.8 . A higher power of magnification allows capturing details of collagen architecture that influence the fractal dimension value and make the difference between certain fibrosis grades. Through this method, a low power magnification offers you only the possibility to identify the presence or the absence of fibrosis.

According to the statistics, the presence or absence of inflammatory cells or necroinflammatory foci in the liver, no matter the degree, does not influence the fractal dimension of an image of liver fibrosis of a certain grade $(p>0.1)$. In the image processing only the collagen fibers are isolated so that if they would be partly covered by inflammatory cells, the architecture of the fibrosis structure would still be identifiable and analyzable.
As others conjectured (Viksna et al., 2012), the histological investigation of liver fibrosis could soon shift from the routine light microscopy to virtual microscopy assisted by digital image analysis (Campos et al., 2014) or completely automated image analysis and staging of the lesions (Calès et al., 2015). The limits of this method, as of any investigation using digital image analysis, are mainly given by the technological logistics, from tissue fixation, processing, the section thickness and staining to the image acquisition mode and the choice of software, that might lead to variations in results across different studies (Losa et al. 2011, Viksna et al., 2012). Nevertheless, the reproducibility of digital image analysis is not affected if the method is identically applied (Campos et al., 2014).

As Dioguardi (2005) said, the "quantitative methods (...) should not be seen as a more sophisticated means of revealing what is observed, but rather as a different way of perceiving it." The present method is a complementary tool to the histological examination, a useful way of digital quantification of fibrosis as it offers a proper measurement of liver fibrosis that can be relevant, especially for the evaluation of an evolving condition.

\section{CONCLUSION}

The images acquired with objective lens $20 \times$ proved to be the most reliable for determining the fractal dimension as the micrographs contained a detailed view over the lesion. The presence of necroinflammatory reaction does not influence the fractal dimension of the collagen tissue architecture. Fractal analysis provides an objective parameter in detecting the presence and the degree of fibrosis over a semiquantitative scoring. The digital processing and the computation of fractal dimension have their settings predetermined, making it a rapid and reproducible method. The method is in its early development phase, as more fibrosis stages need to be analysed, but the study reveals that there is a possibility to define distinct domains for the values of fractal dimension that can be statistically associated with the presence and, respectively, the absence of fibrosis. The estimation of liver fibrosis is complementary to staging liver disease and this technique of fractal analysis can be a useful tool in the initial assessment of proper measurement of fibrosis. 
Acknowledgements. This paper was cofinanced from the European Social Fund through Sectoral Operational Programme - Human Resources Development 2007-2013, project number POSDRU/1871.5/S/155631, entitled "Doctoral programs at the forefront of research excellence in prioritary domains: health, materials, products and innovative processes", Beneficiary - "Carol Davila" University of Medicine and Pharmacy Bucharest.

\section{REFERENCES}

1. Bedossa P, Poynard T (1996). An algorithm for the grading of activity in chronic hepatitis C. The METAVIR Cooperative Study Group. Hepatology, Baltimore, Md., 24:289-293.

2. Calès $P$, Chaigneau J, Hunault $G$, Michalak $S$, CavaroMenard C, Fasquel J-B, Bertrais S, Rousselet M-C (2015). Automated morphometry provides accurate and reproducible virtual staging of liver fibrosis in chronic hepatitis C. Journal of Pathology Informatics, 6: 20

3. Campos CFF, Paiva DD, Perazzo H, Moreira PS, Areco LFF, Terra C, Perez R, Figueiredo FAF (2014). An inexpensive and worldwide available digital image analysis technique for histological fibrosis quantification in chronic hepatitis C. J Viral Hepat 21(3):216-22.

4. Cromey, DW, 2010. Avoiding twisted pixels: ethical guidelines for the appropriate use and manipulation of scientific digital images. Science and engineering ethics, 16(4), pp.639-67.

5. Dioguardi N (2005). Reflections on the quantitative analysis of liver fibrosis in biopsy specimens, p. 411-42. In: Paton R, McNamara L (Eds.). Studies in Multidisciplinarity. Multidisciplinary Approaches to Theory in Medicine. Elsevier.

6. Dioguardi N, Franceschini B, Russo C, Grizzi F (2005). Computer-aided morphometry of liver inflammation in needle biopsies. World J Gastroenterol 11(44):69957000 .
7. Dioguardi N (2011). Hypothesis for a new method to measure the dynamic patterns of tissue injury. Med Hypotheses 77(6):1022-7.

8. Gaiță L, Militaru M, Popescu G (2013). Fractal dimension of chromatin regions in histological pictures reveals the presence of epithelial tumours. Proceedings of the 5th International Conference "Computational Mechanics and Virtual Engineering" COMEC 2013: Transilvania University of Brasov: 209-213.

9. Grizzi F, Russo C, Franceschini B, Di Rocco M, Torri V, Morenghi E, Fassati LR, Dioguardi N. (2006). Sampling variability of computer-aided fractal-corrected measures of liver fibrosis in needle biopsy specimens. World J Gastroenterol 12(47):7660-5.

10. Kukolj V, Aleksić-KovačevićS, Katić-RadivojevićS, Knežević D, Jovanović M (2015). The role and immunophenotypic characteristics of myofibroblasts in liver of sheep naturally infected with the lancet liver fluke (Dicrocoelium dendriticum). Veterinary Parasitology 208(3-4):181-9.

11. Losa GA, Di Ieva A, Grizzi F, De Vico G (2011). On the fractal nature of nervous cell system. Front Neuroanat 5:45

12. Losa GA (2015). The living realm depicted by the fractal geometry. Fractal Geometry and Nonlinear Anal in Med and Biol 1(1):11-15.

13. Viksna L, Strumfa I, Strumfs B, Zalcmane V, Ivanovs A, Sondore V (2012). Future Aspects of Liver Biopsy: From Reality to Mathematical Basis of Virtual Microscopy. p. 257-279. In: Tagaya N. (Ed.). Liver Biopsy - Indications, Procedures, Results. InTech, Croatia

14. Manga-González MY, Ferreras MC, Campo R, GonzálezLanza C, Pérez V, García-Marín JF (2004). Hepatic marker enzymes, biochemical parameters and pathological effects in lambs experimentally infected with Dicrocoelium dendriticum (Digenea). Parasitol Res. 93(5):344-55.

15. Moal F, Chappard D, Wang J, Vuillemin E, Michalak-Provost S, Rousselet MC, Oberti F, Calès P (2002). Fractal dimension can distinguish models and pharmacologic changes in liver fibrosis in rats. Hepatology. 36(4 Pt 1):840-9. 